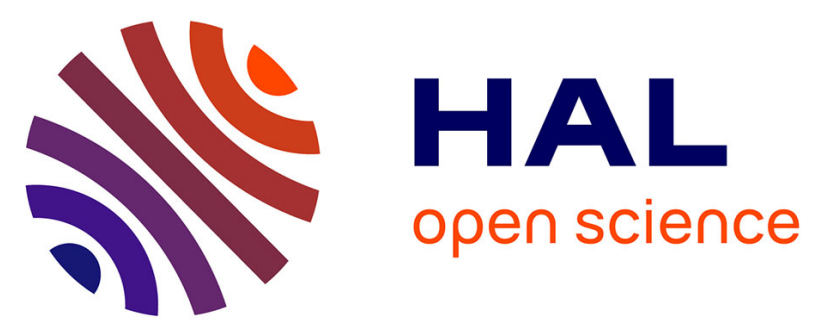

\title{
Boolean CP decomposition of binary tensors: Uniqueness and algorithm
}

Mamadou Diop, Sébastian Miron, Antoine Souloumiac, David Brie

\section{To cite this version:}

Mamadou Diop, Sébastian Miron, Antoine Souloumiac, David Brie. Boolean CP decomposition of binary tensors: Uniqueness and algorithm. 44th IEEE International Conference on Acoustics Speech and Signal Processing, ICASSP 2019, May 2019, Brighton, United Kingdom. 10.1109/ICASSP.2019.8683508 . hal-02314551

\section{HAL Id: hal-02314551 \\ https://hal.science/hal-02314551}

Submitted on 14 Oct 2019

HAL is a multi-disciplinary open access archive for the deposit and dissemination of scientific research documents, whether they are published or not. The documents may come from teaching and research institutions in France or abroad, or from public or private research centers.
L'archive ouverte pluridisciplinaire HAL, est destinée au dépôt et à la diffusion de documents scientifiques de niveau recherche, publiés ou non, émanant des établissements d'enseignement et de recherche français ou étrangers, des laboratoires publics ou privés. 


\title{
BOOLEAN CANONICAL POLYADIC DECOMPOSITION OF BINARY TENSORS: A POST-NONLINEAR MIXTURE MODEL APPROACH
}

\author{
Mamadou Diop*, ${ }^{*}$, Sebastian Miron*, Antoine Souloumiac ${ }^{\ddagger}$ and David Brie ${ }^{*}$ \\ *Université de Lorraine, CRAN, UMR 7039, 54506 Vandoeuvre Cedex, France \\ ${ }^{\dagger}$ CEA Tech Grand-Est, 57075 Metz Technopôle Cedex 3, France \\ ${ }^{\ddagger}$ CEA Saclay, LIST, 91191 Gif-sur-Yvette Cedex, France \\ Email: sebastian.miron@univ-lorraine.fr
}

\begin{abstract}
We propose an algorithm to perform the low-rank Boolean Canonical Polyadic Decomposition (BCPD) of a binary tensor. The proposed approach is based on the AO-ADMM strategy introduced [7] and uses a post-nonlinear mixture model for binary sources. We show that this new method is better adapted for low-rank approximation of binary tensors than other similar methods. We also provide an easy-to-check necessary condition for the uniqueness of the BCPD.
\end{abstract}

Index Terms - binary tensor, Boolean Canonical Polyadic Decomposition, uniqueness, AO-ADMM

\section{INTRODUCTION}

Third-order binary tensors are three-way arrays with the entries composed of 0's and 1's. They are often used to capture ternary relationships, memberships or occurrences of events, e.g., source IP - target IP - target port in network traffic analysis, adjacency matrices of a dynamic graph over time, predicate relations subject - object - verb in knowledge base, etc. To reveal latent structures in these binary tensors, the Boolean Canonical Polyadic Decomposition (BCPD) has been introduced; it allows to decompose a binary-valued tensor in a "logical sum" of rank-1 binary terms (sources) [2]. The BCPD is NP-hard and therefore suboptimal strategies have been proposed to tackle this difficulty. For example, in $[3,4]$ the formal concept analysis framework is used to achieve the decomposition, while in [2], the BCPD is obtained using an alternating approach based on the discrete basis problem for binary matrices, introduced in [5].

All these approaches are based on greedy strategies, designed to give optimal results in the case of exact decompositions and non-correlated sources. Their performance deteriorates rapidly in the presence of binary noise and therefore they are not well suited for low-rank decompositions of binary tensors. In this paper we propose an approach based on

This work was supported by the operational program FEDER-FSE Lorraine et Massif des Vosges 2014-2020. a relaxation of the BCPD problem over the nonnegative real orthant, coupled with a post-nonlinear model of the Boolean mixture of the binary sources. We show that our approach yields very good results for low-rank approximation of binary tensors in the presence of sources having overlapping support (correlated sources). We also proove a sufficient condition for the uniqueness of the BCPD based on relationships between the the support of the loading factors.

\section{BOOLEAN CP DECOMPOSITION OF BINARY TENSORS}

Consider a three-way binary data array (tensor) $\mathcal{X}$ of size $N \times$ $M \times P$ such that its elements $\mathcal{X}_{n m p} \in\{0,1\}$ (with $n=$ $1, \ldots, N, m=1, \ldots, M$ and $p=1, \ldots, P$ ) can be expressed as:

$$
\mathcal{X}_{n m p}=\bigvee_{k=1}^{K}\left(w_{n k} \wedge h_{m k} \wedge v_{p k}\right)
$$

with $w_{n k}, h_{m k}, v_{p k} \in\{0,1\}$, and with " $\vee$ " and " $\wedge$ " denoting the logical "AND" and "OR" operators, respectively. Equation (1) expresses a third-order Boolean Canonical Polyadic Decomposition (BCPD) of rank $K$. As $w_{n k}, h_{m k}, v_{p k} \in$ $\{0,1\}$, the logical "AND" operator in (1) can be equivalently replaced by the classical real numbers product, and thus $\mathcal{X}_{n m p}=\bigvee_{k=1}^{K} w_{n k} h_{m k} v_{p k}$. By regrouping the elements $w_{n k}, h_{m k}$ and $v_{p k}$ on the columns of matrices $\mathbf{W}=\left[\mathbf{w}_{1} \ldots \mathbf{w}_{K}\right](N \times K), \mathbf{H}=\left[\mathbf{h}_{1} \ldots \mathbf{h}_{K}\right](M \times K)$ and $\mathbf{V}=\left[\mathbf{v}_{1} \ldots \mathbf{v}_{K}\right](P \times K)$, respectively, the BCPD (1) can be expressed as:

$$
\mathcal{X}=\bigvee_{k=1}^{K}\left(\mathbf{w}_{k} \circ \mathbf{h}_{k} \circ \mathbf{v}_{k}\right)=\llbracket \mathbf{W}, \mathbf{H}, \mathbf{V} \rrbracket,
$$

where "o" denotes the vector outer product and where the logical operation " $\vee$ " is performed element-wise. Thus, in order to perform the BCPD of $\mathcal{X}$, one must solve the following in- 
verse problem:

$$
\{\hat{\mathbf{W}}, \hat{\mathbf{H}}, \hat{\mathbf{V}}\}=\arg \min _{\mathbf{W}, \mathbf{H}, \mathbf{V} \in\{0,1\}}\left\|\mathcal{X}-\bigvee_{k=1}^{K} \mathbf{w}_{k} \circ \mathbf{h}_{k} \circ \mathbf{v}_{k}\right\|_{2}^{2} .
$$

A classical way to perform the canonical polyadic decomposition in the real-valued case is to alternatingly estimate the three loading matrices using the three $n$-mode unfoldings of $\mathcal{X}$ [1]. A similar strategy can be used in the Boolean case, based on the three unfoldings hereafter:

$$
\begin{aligned}
& \mathbf{X}_{(1)}=\mathbf{W} \diamond(\mathbf{V} \odot \mathbf{H})^{T}, \\
& \mathbf{X}_{(2)}=\mathbf{H} \diamond(\mathbf{V} \odot \mathbf{W})^{T}, \\
& \mathbf{X}_{(3)}=\mathbf{V} \diamond(\mathbf{H} \odot \mathbf{W})^{T},
\end{aligned}
$$

where " $\odot$ " denotes the Khatri-Rao product and " $\diamond$ " represents the Boolean matrix product, i.e., the restriction of BCPD (2) to second-order tensors (matrices).

\section{UNIQUENESS}

Before introducing the proposed approach for performing the BCPD, we analyze the uniqueness of this Boolean tensor decomposition. In [6] we derived a necessary and sufficient uniqueness condition for the Boolean decomposition of binary matrices. An extension of this condition to tensor case is possible but it would not have much practical interest because it would be very difficult to check. We give instead a sufficient condition for the uniqueness of the decomposition (2), much more easy to examine in practice. We start by proving a sufficient condition for the Boolean decomposition of the binary matrix $\mathbf{X}=\mathbf{W} \diamond \mathbf{H}=\bigvee_{k=1}^{K} \mathbf{X}^{(k)}=\bigvee_{k=1}^{K} \mathbf{w}_{k} \mathbf{h}_{k}^{T}$.

Theorem 3.1 (Partial uniqueness of $\mathbf{X}=\mathbf{W} \diamond \mathbf{H})$. If $\operatorname{supp}^{1}\left(\mathbf{w}_{\ell}\right) \nsubseteq \bigcup_{k \neq \ell}^{K} \operatorname{supp}\left(\mathbf{w}_{k}\right)$ then the $\ell^{\text {th }}$ column of $\mathbf{H}$, i.e, $\mathbf{h}_{\ell}$ can be uniquely estimated from $\mathbf{X}$. (A similar condition can be proven for the uniqueness of $\left.\mathrm{w}_{\ell}\right)$.

Proof. Suppose that $\mathbf{h}_{\ell}$ can not be uniquely estimated from $\mathbf{X}$, i.e. it $\exists \overline{\mathbf{X}}^{(\ell)}=\mathbf{w}_{\ell} \overline{\mathbf{h}}_{\ell}^{T} \neq \mathbf{X}^{(\ell)}=\mathbf{w}_{\ell} \mathbf{h}_{\ell}^{T}$ such that $\mathbf{X}=$ $\underset{k \neq \ell}{\vee} \mathbf{w}_{k} \mathbf{h}_{k}^{T} \vee \mathbf{w}_{\ell} \mathbf{h}_{\ell}^{T}=\underset{k \neq \ell}{\vee} \mathbf{w}_{k} \mathbf{h}_{k}^{T} \vee \mathbf{w}_{\ell} \overline{\mathbf{h}}_{\ell}^{T}$. This is equivalent to $\operatorname{supp}(\mathbf{X})=\bigcup_{k=1}^{K} \operatorname{supp}\left(\mathbf{w}_{k} \mathbf{h}_{k}^{T}\right)=\bigcup_{k \neq \ell} \operatorname{supp}\left(\mathbf{w}_{k} \mathbf{h}_{k}^{T}\right) \cup \operatorname{supp}\left(\mathbf{w}_{\ell} \mathbf{h}_{\ell}^{T}\right)$ $=\cup_{k \neq \ell} \operatorname{supp}\left(\mathbf{w}_{k} \mathbf{h}_{k}^{T}\right) \cup \operatorname{supp}\left(\mathbf{w}_{\ell} \overline{\mathbf{h}}_{\ell}^{T}\right)$. Let us suppose, without loss of generality, that $\overline{\mathbf{h}}_{\ell}=\mathbf{h}_{\ell} \vee \mathbf{h} \neq \mathbf{h}_{\ell}$. Then, $\operatorname{supp}(\mathbf{X})=$ $\cup_{k \neq \ell} \operatorname{supp}\left(\mathbf{w}_{k} \mathbf{h}_{k}^{T}\right) \cup \operatorname{supp}\left(\mathbf{w}_{\ell}\left(\mathbf{h}_{\ell} \vee \mathbf{h}\right)^{T}\right)=\cup_{k \neq \ell} \operatorname{supp}\left(\mathbf{w}_{k} \mathbf{h}_{k}^{T}\right) \cup$ $\operatorname{supp}\left(\mathbf{w}_{\ell} \mathbf{h}_{\ell}^{T}\right) \cup \operatorname{supp}\left(\mathbf{w}_{\ell} \mathbf{h}^{T}\right)$. As $\operatorname{supp}(\mathbf{h}) \nsubseteq \operatorname{supp}\left(\mathbf{h}_{\ell}\right)$, it results that $\operatorname{supp}\left(\mathbf{w}_{\ell} \mathbf{h}^{T}\right) \subseteq \bigcup_{k \neq \ell} \operatorname{supp}\left(\mathbf{w}_{k} \mathbf{h}_{k}^{T}\right) \Leftrightarrow \operatorname{supp}\left(\mathbf{w}_{\ell}\right) \subseteq$ $\cup_{k \neq \ell} \operatorname{supp}\left(\mathbf{w}_{k}\right)$ and $\operatorname{supp}(\mathbf{h}) \subseteq \underset{k \neq \ell}{\cup} \operatorname{supp}\left(\mathbf{h}_{k}\right)$, which ends the proof.

\footnotetext{
${ }^{1}$ We define the support of a vector $\mathbf{x}$ as $\operatorname{supp}(\mathbf{x})=\left\{i, \mathbf{x}_{i} \neq 0\right\}$ and the support of matrix $\mathbf{X}$ as $\operatorname{supp}(\mathbf{X})=\left\{(i, j), \mathbf{X}_{i j} \neq 0\right\}$.
}

By applying theorem 3.1 to the unfoldings $\mathbf{X}_{(1)}, \mathbf{X}_{(2)}$ and $\mathbf{X}_{(3)}$ of $\mathcal{X}$, the following partial uniqueness condition for BCPD can be proven quite straightforwardly (for space reasons the proof will not be detailed in this version of the paper).

Theorem 3.2 (Partial uniqueness of the BCPD of $\mathcal{X}$ ). The $\ell^{\text {th }}$ rank-1 term $\mathcal{X}^{(\ell)}=\mathbf{w}_{\ell} \circ \mathbf{h}_{\ell} \circ \mathbf{v}_{\ell}$ in the BCPD (2) can be uniquely estimated from $\mathcal{X}$ if

$$
\begin{aligned}
& \operatorname{supp}\left(\mathbf{w}_{\ell}\right) \nsubseteq \bigcup_{k \neq \ell}^{K} \operatorname{supp}\left(\mathbf{w}_{k}\right) \text { and } \operatorname{supp}\left(\mathbf{h}_{\ell}\right) \nsubseteq \bigcup_{k \neq \ell}^{K} \operatorname{supp}\left(\mathbf{h}_{k}\right) \\
& \operatorname{or} \\
& \operatorname{supp}\left(\mathbf{w}_{\ell}\right) \nsubseteq \bigcup_{k \neq \ell}^{K} \operatorname{supp}\left(\mathbf{w}_{k}\right) \text { and } \operatorname{supp}\left(\mathbf{v}_{\ell}\right) \nsubseteq \bigcup_{k \neq \ell}^{K} \operatorname{supp}\left(\mathbf{v}_{k}\right) \\
& \operatorname{supp}\left(\mathbf{h}_{\ell}\right) \nsubseteq \bigcup_{k \neq \ell}^{K} \operatorname{supp}\left(\mathbf{h}_{k}\right) \text { and } \operatorname{supp}\left(\mathbf{v}_{\ell}\right) \nsubseteq \bigcup_{k \neq \ell}^{K} \operatorname{supp}\left(\mathbf{v}_{k}\right) .
\end{aligned}
$$

If theorem 3.2 is satisfied for all values of $\ell=1, \ldots, K$, then the BPCD is fully unique or more simply we say that the $\mathrm{BPCD}$ is unique.

\section{PROPOSED APPROACH}

We base our algorithm for solving the BCPD problem (3) on a post-nonlinear mixture model approach, similar to the one that we proposed in [6] for the matrix case. Instead of solving directly (3), we solve a relaxed version of it :

$\{\hat{\mathbf{W}}, \hat{\mathbf{H}}, \hat{\mathbf{V}}\}=\arg \min _{\mathbf{W}, \mathbf{H}, \mathbf{V} \in\{0,1\}}\left\|\mathcal{X}-\Phi\left(\sum_{k=1}^{K} \mathbf{w}_{k} \circ \mathbf{h}_{k} \circ \mathbf{v}_{k}\right)\right\|_{2}^{2}$,

where $\Phi(x)$ is the sigmoid function in figure 1 applied element-wise.

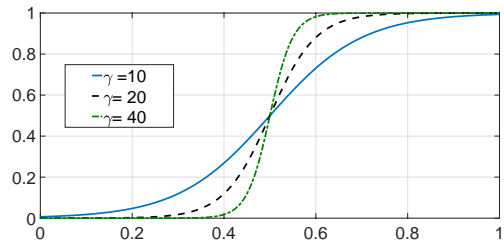

Fig. 1: Sigmoid function $\Phi(x)=\frac{1}{1+e^{-\gamma(x-0.5)}}$

In this paper we develop an algorithm for solving (7) inspired from the Alternating Optimization - Alternating Direction Method of Multipliers (AO-ADMM) introduced in [7]. The proposed approach can be summarized as follows:

\section{Repeat}

$\min _{\mathbf{W}, \overline{\mathbf{W}}} \frac{1}{2}\left\|\mathbf{X}_{(1)}-\Phi\left(\overline{\mathbf{W}}(\mathbf{V} \odot \mathbf{H})^{T}\right)\right\|_{F}^{2}+\|\mathbf{W}-\mathbf{W} * \mathbf{W}\|_{F}^{2}$ subject to $\mathbf{W}=\overline{\mathbf{W}}$ 


$$
\min _{\mathbf{H}, \overline{\mathbf{H}}} \frac{1}{2}\left\|\mathbf{X}_{(2)}-\Phi\left(\overline{\mathbf{H}}(\mathbf{V} \odot \mathbf{W})^{T}\right)\right\|_{F}^{2}+\|\mathbf{H}-\mathbf{H} * \mathbf{H}\|_{F}^{2}
$$

subject to $\mathbf{H}=\overline{\mathbf{H}}$

$$
\begin{aligned}
& \min _{\mathbf{V}, \overline{\mathbf{V}}} \frac{1}{2}\left\|\mathbf{X}_{(3)}-\Phi\left(\overline{\mathbf{V}}(\mathbf{H} \odot \mathbf{W})^{T}\right)\right\|_{F}^{2}+\|\mathbf{V}-\mathbf{V} * \mathbf{V}\|_{F}^{2} \\
& \text { subject to } \mathbf{V}=\overline{\mathbf{V}} \\
& \text { until convergence }
\end{aligned}
$$

where " $*$ " denotes the matrix Hadamard (element-wise) product. The second term in the expressions to minimize is used to constraint the entries of $\mathbf{W}, \mathbf{H}, \mathbf{W}$ to binarity, as explained in [6]. Using the results of [7], update rules can be obtained for the three minimization problems. For example, for the update of $\mathbf{W}$, the following expressions are obtained:

$$
\begin{aligned}
& \mathbf{\mathbf { W }} \leftarrow \underset{\overline{\mathbf{W}}}{\arg \min } \frac{1}{2}\left\|\mathbf{X}_{(1)}-\Phi\left(\overline{\mathbf{W}}(\mathbf{V} \odot \mathbf{H})^{T}\right)\right\|_{F}^{2}+ \\
& \qquad \frac{\rho}{2}\|\mathbf{W}-\overline{\mathbf{W}}+\mathbf{A}\|_{F}^{2} \\
& \mathbf{W} \leftarrow \underset{\mathbf{W}}{\arg \min }\|\mathbf{W}-\mathbf{W} * \mathbf{W}\|_{F}^{2}+\frac{\rho}{2}\|\mathbf{W}-\overline{\mathbf{W}}+\mathbf{A}\|_{F}^{2} \\
& \mathbf{A} \leftarrow \mathbf{A}+\mathbf{W}-\mathbf{W},
\end{aligned}
$$

with $\rho$ a regularization parameter. One can easily observe that the two minimization problems below are non-convex. In order to obtain an explicit update rule for $\mathbf{W}$, we take advantage of the binary nature of its entries and we replace the term $\|\mathbf{W}-\mathbf{W} * \mathbf{W}\|_{F}^{2}$ by $\|\mathbf{W}-\mathbf{W} * \mathbf{W}\|_{1}$. For $\overline{\mathbf{W}}$, we locally "linearize" the sigmoid function $\Phi(\cdot)$, in the neighborhood of the current iteration of $\overline{\mathbf{W}}$. The resulting algorithm, that we called Boolean Tensor - ADMM (BT-ADMM), is resumed in Algorithm 1.

\section{RESULTS}

In this section we illustrate the proposed approach in numerical simulations and compare it to similar methods of the stateof-the-art.

A first experiment illustrates the uniqueness condition for the BCPD. Figure 2 shows two rank-3 BCPD's of $10 \times 7 \times 5$ binary tensors (gray pixels symbolize the 1's). The first row of each image represents the simulated data and the second row, the estimated BCPD. One can see that for Fig.2 $(a)$ the uniqueness conditions of theorem 3.2 are verified for all 3 sources, while for Fig.2 (b) the partial uniqueness condition is not satisfied for the second source. Thus, for the second configuration, our algorithm yielded another decomposition that reproduces exactly the BCP model $\mathcal{X}$.

The second experiments compares the performance of the proposed BT-ADMM algorithm to two other state-of-the-art approaches, the BCP-ALS of [2] and the T-FC of [4]. We

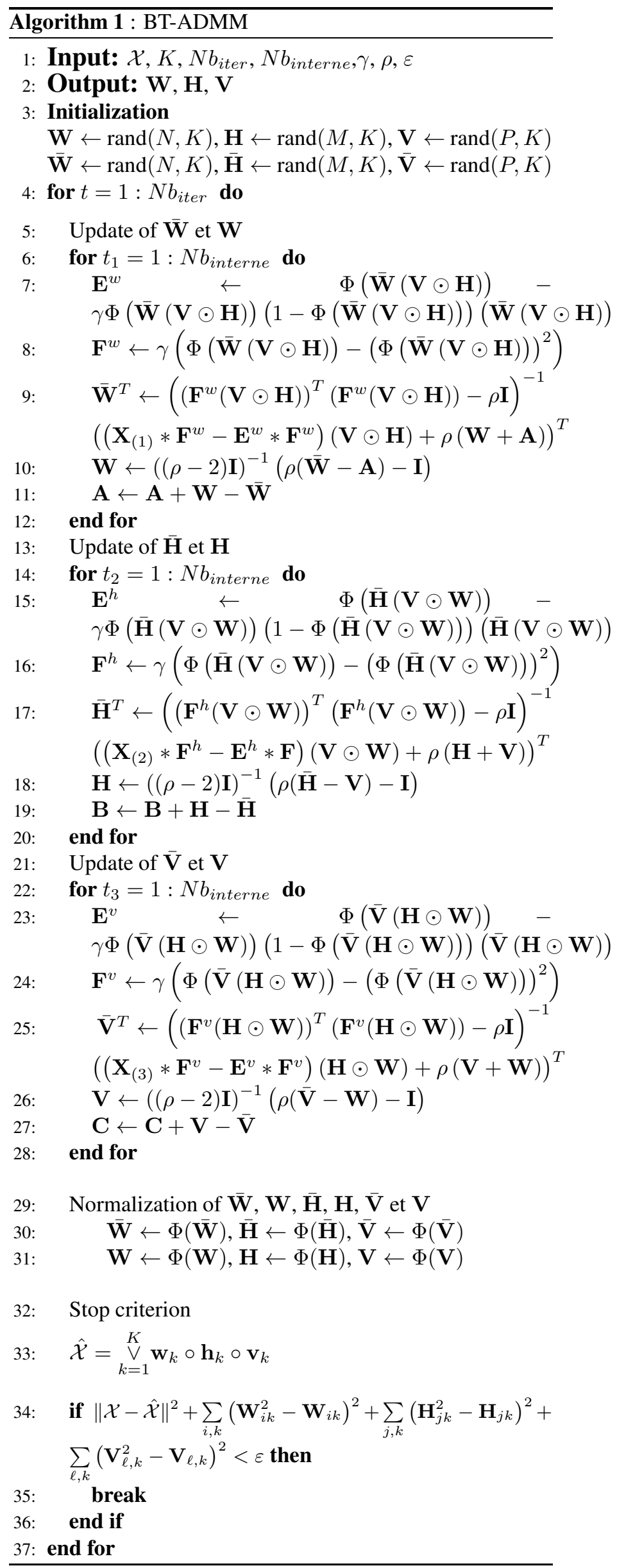



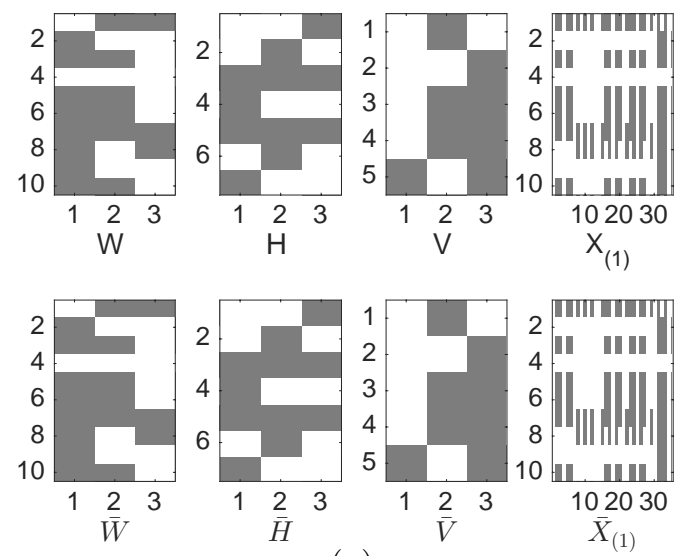

$(a)$
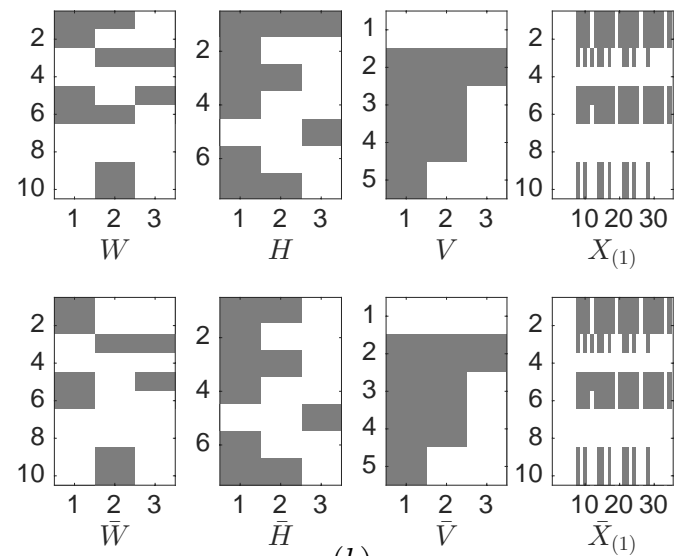

(b)

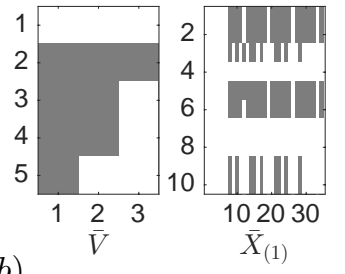

Fig. 2: Unique decomposition $(a)$ and non-unique decomposition (b) of $\mathcal{X}$

plotted the estimation error for the loading matrices:

$$
\operatorname{Error}_{\mathbf{W}, \mathbf{H}, \mathbf{V}}=\frac{\frac{\|\mathbf{W}-\hat{\mathbf{W}}\|_{F}^{2}}{N K}+\frac{\|\mathbf{H}-\hat{\mathbf{H}}\|_{F}^{2}}{M K}+\frac{\|\mathbf{V}-\hat{\mathbf{V}}\|_{F}^{2}}{P K}}{3}
$$

and the reconstruction error for $\mathcal{X}$ :

$$
\operatorname{Error}_{\mathcal{X}}=\frac{\left\|\mathbf{X}_{(1)}-\hat{\mathbf{X}}_{(1)}\right\|_{F}^{2}}{N M P}
$$

versus the noise rate. For these simulations we considered additive XOR noise generated according to a Bernoulli distribution of parameter $b$. The plotted points were averaged over 50 runs. Two scenarios are considered: in the first scenario (Fig. 3) the sources are randomly simulated according to a Bernoulli distribution with parameter $p=0.3$, i.e., the sources have low correlation (their supports are approximately disjoints). In the second scenario (Fig. 4) we chose $p=0.6$ in order to generate highly correlated sources.

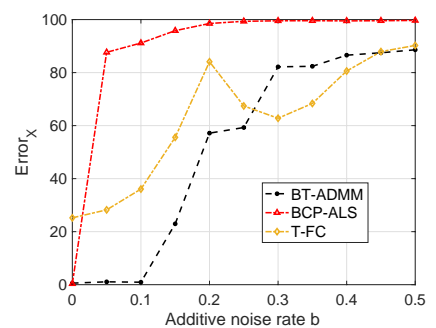

(a)

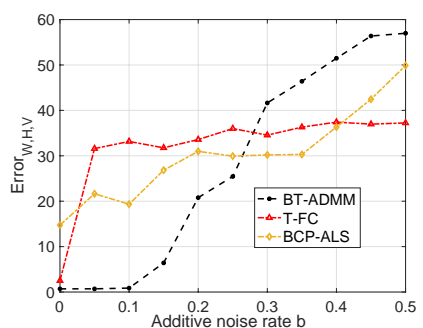

(b)
Fig. 3: (a) Reconstruction error rate for $\mathcal{X}$ and (b) estimation error rate for $\mathbf{W}, \mathbf{H} \mathbf{V}$ vs. additive XOR noise rate $b(N=20, M=30$, $P=10, K=3, \rho=10^{9}$ ) for $p=0.3$

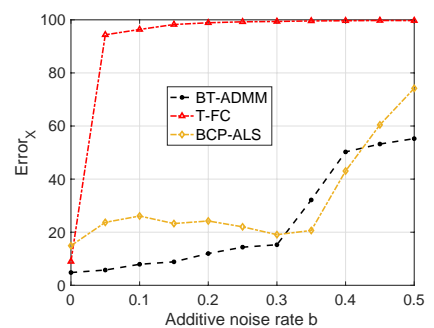

(a)

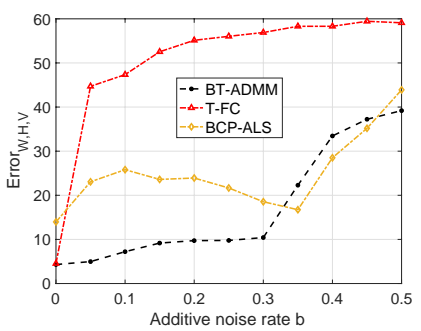

(b)
Fig. 4: (a) Reconstruction error rate for $\mathcal{X}$ and (b) estimation error rate for $\mathbf{W}, \mathbf{H} \mathbf{V}$ vs. additive XOR noise rate $b(N=20, M=30$, $P=10, K=3, \rho=10^{9}$ ) for $p=0.6$

One can observe that for noise rates $b$ inferior to 0.3 our algorithm yields better results than the competitor methods, which makes it an interesting tool for low-rank binary tensor approximation. For the values of $p>0.3$ none of the three methods give good results because the XOR noise rate is high enough to completely destroy the low-rank structure of the data.

\section{CONCLUSIONS}

In this paper we introduced a new method for the Boolean canonical polyadic decomposition (BCPD) of binary-valued tensors based on a post-nonlinear mixture model and an alternating ADMM approach. We illustrated in numerical simulations that our method outperforms similar state-of-the-art methods in the presence of XOR binary noise, which makes it well-adapted for low-rank binary tensor approximation. An easy-to-check sufficient condition for the BCPD was also derived. 


\section{REFERENCES}

[1] Tamara G. Kolda and Brett W. Bader, "Tensor decompositions and applications," SIAM review, vol. 51, no. 3, pp. 455-500, 2009.

[2] Pauli Miettinen, "Boolean tensor factorizations," in IEEE 11th International Conference on Data Mining (ICDM 2011). IEEE, 2011, pp. 447-456.

[3] Radim Belohlavek and Vilem Vychodil, "Optimal factorization of three-way binary data," in IEEE International Conference on Granular Computing ( $\mathrm{GrC}$ 2010). IEEE, 2010, pp. 61-66.

[4] Radim Belohlavek, Cynthia Glodeanu, and Vilem Vychodil, "Optimal factorization of three-way binary data using triadic concepts," Order, vol. 30, no. 2, pp. 437454, 2013.

[5] Pauli Miettinen, Taneli Mielikainen, Aristides Gionis, Gautam Das, and Heikki Mannila, "The discrete basis problem," IEEE Transactions on Knowledge and Data Engineering, vol. 20, no. 10, pp. 1348-1362, 2008.

[6] Mamadou Diop, Anthony Larue, Sebastian Miron, and David Brie, "A post-nonlinear mixture model approach to binary matrix factorization," in 25th European Signal Processing Conference (EUSIPCO 2017). IEEE, 2017, pp. 321-325.

[7] Kejun Huang, Nicholas D. Sidiropoulos, and Athanasios P. Liavas, "A flexible and efficient algorithmic framework for constrained matrix and tensor factorization," IEEE Transactions on Signal Processing, vol. 64, no. 19, pp. 5052-5065, 2016. 